\title{
In silico Analysis of Flavonoid Glycosides and its Aglycones as Reverse Transcriptase Inhibitor
}

\author{
Stefandi J Wijaya, Arry Yanuar, Rosita Handayani, Rezi Riadhi Syahdi*
}

Stefandi J Wijaya, Arry Yanuar, Rosita Handayani, Rezi Riadhi Syahdi*

Faculty of Pharmacy, Universitas Indonesia, Depok, 16424, INDONESIA.

\section{Correspondence}

Rezi Riadhi Syahdi

Faculty of Pharmacy, University of Indonesia, Depok, INDONESIA.

E-mail: rezi.riadhi@farmasi.ui.ac.id

History

- Submission Date: 31-01-2019;

- Review completed: 28-04-2019;

- Accepted Date: 24-05-2019.

DOI : 10.5530/pj.2019.11.194

Article Available online http://www.phcogj.com/v11/i6

\section{Copyright}

(C) 2019 Phcogj.Com. This is an openaccess article distributed under the terms of the Creative Commons Attribution 4.0 International license.

\section{ABSTRACT}

Background: HIV continues to be a major global public health issue, having claimed more than 35 million lives so far. In 2016, 1 million people died from HIV-related causes globally. HIV-1 reverse transcriptase is one of HIV's vital enzymes for virus reproduction. If the enzyme is inhibited, the virus multiplication could be significantly decreased. There are currently many treatments for HIV, but more effective treatment is always needed because of the possibility of drug resistance and side effects for long-term use. Based on the previous study, there are some natural compounds with high affinity to the HIV-1 reverse transcriptase enzyme. Some of these compounds are flavonoid glycosides. Aims and Method: This study was aimed to learn more about in silico HIV-1 reverse transcriptase inhibitory activities of flavonoid glycosides using docking method. Results: The results showed that the most recommended flavonoid glycosides are those with the lowest binding energy, which were kaempferol-3-O-rhamnoside, myricetin-3-O-rhamnoside and quercetin-3-O-rhamnoside. This was due to the interactions of all three flavonoid rings and sugar moiety with key amino acid residues, which were Leu100, Lys101, Glu138, Tyr181, His235 and Tyr318. Conclusion: Flavonoid glycosides with rhamnose as glycone showed lower binding energy on HIV-1 reverse transcriptase.

Key words: Flavonoid, Glycosides, HIV, Molecular docking, Reverse transcriptase.

\section{INTRODUCTION}

Human Immunodeficiency Virus (HIV) is a virus that destructs and impairs the function of immune cells. ${ }^{1}$ Like any other viruses, it needs to replicate to survive. When replicating in the host cell, HIV utilizes vital production enzymes, i.e. reverse transcriptase, protease, integrase to create mature virions. HIV contains RNA genomic in its virion and within the host cell, it will be converted by reverse transcriptase to cDNA (complementary DNA) which in turn will be integrated into the host cell to produce the essential protein for new viral maturation. Because of RT essential role in the HIV life cycle, it has been considered as major antiHIV-1 drug targets. ${ }^{2}$ As HIV multiplies in the body, the virus sometimes mutates (changes form) and produces variations itself. Variations of HIV that develop while a person is taking HIV medicines can lead to drug-resistant strains of HIV. ${ }^{3}$ There are currently many treatments for HIV, however more effective treatment is always needed because of the possibility of drug resistance and side effects for long-term use. ${ }^{4}$ Secondary metabolites from natural products have been the most successful source of potential drug leads. ${ }^{5}$ Based on the previous study, there are some natural compounds with high affinity to the HIV-1 reverse transcriptase enzyme and some of these compounds are flavonoid glycosides. Glycoside is a compound that consists of a simple sugar (glycone) and another compound (aglycone) via the glycosidic bond. Glycosylation in flavonoids shows a various biological effect, yet it is very difficult to generalize. ${ }^{6}$ It is not easy and time- consuming to learn more about the glycosylation effects on flavonoid glycosides. In silico methods have been widely applied to pharmacology hypothesis development and testing. In silico study could minimize failure possibilities in tests conducted in vivo or in vitro by predicting failure possibilities such as the interaction between a compound with target receptors. Therefore, in silico method was used.? This study was aimed to give a recommendation of compounds that have the potential to be HIV-1 reverse transcriptase inhibitor.

\section{METHODS AND MATERIALS}

\section{Hardware}

A server computer with following specifications: processor (CPU) Intel ${ }^{\circ}$ Core $^{\text {tux }}$ i7 $950 @ 3.07 \mathrm{GHz}$ graphics processing unit (GPU) Nvidia ${ }^{\star}$ GeForce GTX 680 and 24 GB Random Access Memory (RAM) DDR3 with Windows 10 Pro was used. A client computer with the following specification: processor (CPU) Intel ${ }^{\oplus} \mathrm{Core}^{\mathrm{mm}}$ i7 $7700 \mathrm{HQ}$, graphics processing unit (GPU) Nvidia ${ }^{\circledR}$ GeForce GTX 1050 and 8 GB RAM DDR4 with Windows 10 Pro was used.

\section{Software}

The software used in this research are PyMOL (DeLano Scientific LLC, Italy), AutoDockTools (The Scripps Research Institute, USA), VEGAZZ, LigandScout (InteLigand, Austria), MarvinSketch (ChemAxon, Budapest), LigPlot (EMBL-EBI, UK), dan PyRx (The Scripps Research Institute, USA). 


\section{D structure}

The flavonoid glycosides and its aglycones [aglycones: kaempferol, myricetin and quercetin; glycones: arabinose, galactose, glucose, rhamnose and rutinose], macromolecule compounds HIV-1 reverse transcriptase (3LP1) and positive control compound (Figure 1).

\section{Preparation of macromolecule structure}

The preparation process consisted of searching and downloading, optimization and separation of non-standard residues.

\section{Preparation of ligand 3D structure}

There were two types of ligands used for docking, i.e. (1) Flavonoid glycosides and its aglycones available in ChemSpider Search and Share Chemistry (http://ChemSpider.com), PubChem Open Chemistry Database (http://PubChem.ncbi.nlm.gov) and other sources; (2) Positive controls obtained from literature studies which activities had been recognized by the FDA for HIV treatment and available in the PubChem Open Chemistry Database (http://PubChem.ncbi.nlm.gov) and ChemSpider. After that, those ligands were prepared and optimized using VEGAZZ. Preparation and optimization were done by adding hydrogen, fixing charges and minimization.

\section{Molecular docking of positive control and flavonoid glycosides compound}

The docking of positive control compounds was performed to obtain the score of each active compound used for docking. The positive controls used were nevirapine and efavirenz which activities had been recognized by the FDA for HIV treatment. Docking of positive control against HIV-1 reverse transcriptase macromolecule was conducted by molecular docking method. Molecular docking was performed using AutoDock4. The coordinates of the binding site were $\mathrm{x}=10.350 ; \mathrm{y}=$ 14.076; $\mathrm{z}=18.252$ using $50 \times 50 \times 50$ unit grid box with spacing per unit of 0.375 angstrom. AutoDock 4 was run with set parameters: number generation algorithm 27,000, calculate 2,500,000 times (Medium), population 150 and the implementation of running GA as much as 10 times. The results of molecular docking saved as ${ }^{\star}$.dlg file format.

\section{Analysis and visualization of protein-ligand interaction}

The results of docking by using AutoDock4 were first converted to *.pdbqt using AutoDockTools. The visualization of docking results was performed using LigandScout, LigPlot dan PyMOL software.

The purpose of the analysis and visualization was to analyze the presence of hydrogen bond, hydrophobic interaction between ligand compound and positive control to each targetted receptor.

\section{RESULTS}

The docking was run with AutoDock4 parameters. The parameters used were the number of algorithm generation of 27,000 , population of 150, calculations as much as 2,500,000 times (medium) and the implementation of running GA as much as 10 times. This method had been optimized by previous research. ${ }^{2}$ The results were shown in Table 1.<smiles></smiles>

Quercetin<smiles></smiles>

Kaempferol<smiles>O=c1c(O)c(-c2cc(O)c(O)c(O)c2)oc2cc(O)cc(O)c12</smiles>

Myricetin

Figure 1: Structure of Quercetin, Kaempferol and Myricetin ( $R$ = sugar moiety).

Table 1: Molecular docking binding affinity of flavonoid glycosides and aglycones, ranked by lowest binding free energy. Efavirenz and nevirapine were used as NNRTI positive control.

\begin{tabular}{ccc}
\hline No & Ligands & Binding Free Energy/ $\Delta \mathrm{G}(\mathrm{kcal} / \mathrm{mol})$ \\
\hline 1 & Kaempferol-3-O-rhamnoside & -9.04 \\
2 & Efavirenz & -8.92 \\
3 & Myricetin-3-O-rhamnoside & -8.74 \\
4 & Quercetin-3-O-rhamnoside & -8.74 \\
5 & Kaempferol-3-O-arabinoside & -8.7 \\
6 & Quercetin & -8.28 \\
7 & Kaempferol-3-O-glucoside & -8.27 \\
8 & Quercetin 3-O-arabinoside & -8.24 \\
9 & Kaempferol & -8.13 \\
10 & Myricetin & -8.13 \\
11 & Myricetin-3-O-galactoside & -7.85 \\
12 & Nevirapine & -7.75 \\
13 & Quercetin-3-O-galactoside & -7.74 \\
14 & Quercetin-3-O-glucoside & -7.74 \\
15 & Myricetin-3-O-arabinoside & -7.73 \\
16 & Kaempferol-3-O-galactoside & -7.7 \\
17 & Myricetin-3-O-glucoside & -7.42 \\
18 & Quercetin-3-O-rutinoside & -5.43 \\
19 & Kaempferol-3-O-rutinoside & -5.35 \\
20 & Myricetin 3-O-rutinoside & -3.94 \\
\hline
\end{tabular}

[*positive control] 
The positive control that had the lowest binding free energy $-8.92 \mathrm{kcal} /$ mol was efavirenz. Flavonoid glycosides with rhamnose as aglycone indicated lower binding energy.

\section{DISCUSSION}

In this study, there were three types of aglycones and five types of sugar used. Based on the previous study, quercetin as glycoside had good affinity against HIV-1 reverse transcriptase. ${ }^{2}$ Therefore, for comparison, other flavonoids with similar structures were needed. Kaempferol and myricetin were chosen. Arabinoside, galactoside, glucoside and rhamnoside were chosen because of their common presence in nature. Two NNRTI drugs; efavirenz and nevirapine, were chosen because of their good affinity that was exhibited in the previous study. ${ }^{2}$

From this research, flavonoid glycosides with rhamnoside as the glycone group indicated lower binding free energy than the other glycosides and also its aglycones. This result is in line with the previous in vitro study by Behbahani (2014) which showed that kaempferol-7-Oglucoside had a better anti-HIV1 activity than its aglycone. ${ }^{8}$

Rutinoside glycosides showed higher binding free energy than the other glycosides and its aglycones. It signified that disaccharide in flavonoid glycosides might decrease its affinity with HIV-1 reverse transcriptase. The reason of rutinoside glycosides had higher binding free energy was because of its interaction with amino acid residues not as good as rhamnose glycosides. As shown in Figure 2 and Table 2 , all of the rhamnose glycosides had interactions with Tyr181 residues which was one of the key amino acids in HIV-1 reverse transcriptase. ${ }^{9}$ Furthermore, the location where amino acid residues are bound also affects binding free energy value. All of the flavonoid rings and also sugar moiety must interact with amino acid residues to have a lower binding energy.

The Protein-ligand interaction was analyzed and visualized using LigandScout, LigPlot and PyMOL ${ }^{10-12}$ to inspect the hydrogen bond distance and hydrophobic interactions (Table 2).
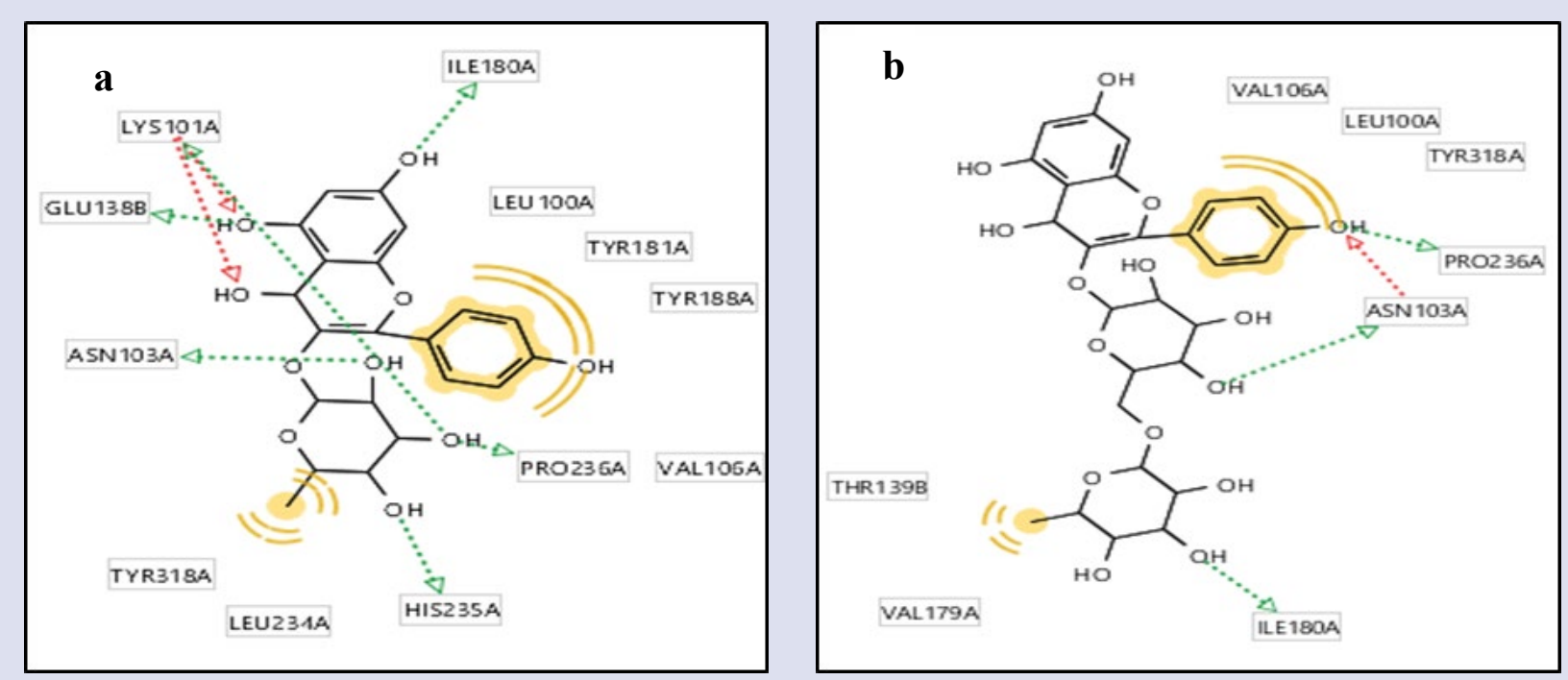

Figure 2: Visualization of (a) Kaempferol-3-O-Rhamnoside; (b) Kaempferol-3-O-Rutinoside.

Table 2: Ligands and amino acid residues interaction. Hydrogen bonds distance were given, hydrophobic or van der walls interactions were indicated by tick $(\sqrt{ })$.

\begin{tabular}{|c|c|c|c|c|c|c|c|c|c|c|c|c|c|c|c|}
\hline Compound & Leu100 & Lys101 & Asn 103 & Val106 & Glu138 & Thr139 & Val179 & Ile180 & Tyr181 & Tyr188 & Trp229 & Leu234 & His235 & Pro236 & Tyr318 \\
\hline Kaempferol & $\checkmark$ & $2.62 \AA$ & - & $\checkmark$ & $3.04 \AA$ & - & - & $2.85 \AA$ & - & - & - & $\checkmark$ & - & - & - \\
\hline Arabinoside & $\checkmark$ & $3.0 \AA$ & $2.72 \AA$ & - & - & - & - & - & $\checkmark$ & $\checkmark$ & $\checkmark$ & - & $2.61 \AA$ & - & $3.0 \AA$ \\
\hline Galactoside & $\checkmark$ & $2.75 \AA$ & - & $\checkmark$ & $2.69 \AA$ & - & - & - & - & - & - & - & - & - & $3.4 \AA$ \\
\hline Glucoside & $\checkmark$ & $2.73 \AA$ & - & - & - & - & - & - & $\checkmark$ & $\checkmark$ & $\checkmark$ & $3.5 \AA$ & - & - & $2.58 \AA$ \\
\hline Rhamnoside & $\checkmark$ & $2.62 \AA$ & $2.69 \AA$ & $\checkmark$ & $2.98 \AA$ & - & - & $2.94 \AA$ & $\checkmark$ & $\checkmark$ & - & $\checkmark$ & $3.28 \AA$ & $3.4 \AA$ & $\checkmark$ \\
\hline Rutinoside & $\checkmark$ & - & $2.41 \AA$ & $\checkmark$ & - & $\checkmark$ & $\checkmark$ & $3.72 \AA$ & - & - & - & - & - & $2.41 \AA$ & $\checkmark$ \\
\hline Myricetin & - & $2.95 \AA$ & - & - & $2.8 \AA$ & - & - & - & - & - & - & - & - & - & $2.9 \AA$ \\
\hline Arabinoside & - & $3.27 \AA$ & $3.0 \AA$ & - & - & - & - & - & - & - & - & - & - & - & $2.7 \AA$ \\
\hline Galactoside & - & $2.8 \AA$ & $2.69 \AA$ & - & - & - & - & - & - & - & - & - & - & $2.9 \AA$ & - \\
\hline Glucoside & - & $2.71 \AA$ & - & - & $2.64 \AA$ & - & - & - & - & - & - & - & - & $3.16 \AA$ & $3.06 \AA$ \\
\hline Rhamnoside & $\checkmark$ & $2.95 \AA$ & - & - & $2.68 \AA$ & - & - & - & $\checkmark$ & - & - & - & $2.19 \AA$ & $2.92 \AA$ & $2.76 \AA$ \\
\hline Rutinoside & $\checkmark$ & $2.67 \AA$ & $2.73 \AA$ & - & - & - & $\checkmark$ & - & - & $2.38 \AA$ & - & - & - & - & - \\
\hline Quercetin & $\checkmark$ & $3.04 \AA$ & - & - & $\checkmark$ & - & $\checkmark$ & - & - & - & - & - & - & - & $2.9 \AA$ \\
\hline Arabinoside & $\checkmark$ & $2.97 \AA$ & $2.9 \AA$ & - & - & - & - & - & $\checkmark$ & $\checkmark$ & - & - & $2.6 \AA$ & - & $3.1 \AA$ \\
\hline Galactoside & $\checkmark$ & $3.06 \AA$ & - & $\checkmark$ & $2.62 \AA$ & - & - & - & - & - & - & - & $3.7 \AA$ & - & $2.91 \AA$ \\
\hline Glucoside & $\checkmark$ & $2.86 \AA$ & - & $\checkmark$ & $2.61 \AA$ & - & - & - & - & - & - & - & - & - & $3.03 \AA$ \\
\hline Rhamnoside & $\checkmark$ & $3.02 \AA$ & - & $\checkmark$ & $2.63 \AA$ & - & - & - & $\checkmark$ & - & - & - & $2.35 \AA$ & - & $3.02 \AA$ \\
\hline Rutinoside & $\checkmark$ & $2.67 \AA$ & $2.47 \AA$ & $\checkmark$ & $2.96 \AA$ & - & $\checkmark$ & - & - & $2.82 \AA$ & - & - & - & $2.51 \AA$ & $\checkmark$ \\
\hline
\end{tabular}




\section{CONCLUSIONS}

Flavonoid glycosides with rhamnose as glycone showed lower binding free energy on HIV-1 Reverse Transcriptase, compared to other sugar moiety and to the aglycones. Monosaccharide as glycone moiety exhibited better binding affinity compared to disaccharides. This result need to be explored further by in vitro and/or in vivo experiment.

\section{ACKNOWLEDGMENT}

The authors would like to thanks DRPM UI for the funding of the research. This paper and research was funded by PITTA 2018 (Publikasi Terindeks International untuk Tugas Akhir Mahasiswa).

\section{REFERENCES}

1. WHO. HIV/AIDS [Internet]. WHO;2018. http://www.who.int/news-room/factsheets/detail/hiv-aids.

2. Syahdi RR, Mun'im A, Suhartanto H, Yanuar A. Virtual screening of Indonesian herbal database as HIV-1 reverse transcriptase inhibitor. Bioinformation. 2003;8(24):1206-10.

3. AIDSInfo. Drug Resistance [Internet]. U.S. Department of health and human services;2018. https://aidsinfo.nih.gov/understanding-hiv-aids/factsheets/21/56/drug-resistance.
4. Zhan P, Pannecouque C, Clercq ED, Liu X. Anti-HIV drug discovery and development: Current innovations and future trends. Journal of Medicinal Chemistry. 2015;59(7):2849-78.

5. Dias DA, Urban S, Roessner U. A historical overview of natural products in drug discovery. Metabolites. 2012;2:303-36.

6. Xiao J. Dietary flavonoid aglycones and their glycosides: Which show better biological significance? Critical Reviews in Food Science and Nutrition. 2017;57(9):1874-905.

7. Ekins S, Mestres J, Testa B. In silico pharmacology for drug discovery: Methods for virtual ligand screening and profiling. Br J Pharmacol. 2007;159:9-20.

8. Behbahani M, Sayedipour S, Pourazar A, Shanehsazzadeh M. In vitro anti-HIV-1 activities of kaempferol and kaempferol-7-O-glucoside isolated from Securigera securidaca. Res Pharm Sci. 2014;9(6):463-9.

9. Delano WL. PyMOL User Guide;2004. http://pymol.sourceforge.net/newman/ userman.pdf/.

10. Wolber G. LigandScout User Manual;2010. http://www.inteligand.com/ ligandscout3/downloads/ligandscout-manual-2010-04-15.pdf

11. Wallace AC, Laskowski RA, Thornton JM. LIGPLOT: A program to generate schematic diagrams of protein-ligand interactions. Protein Eng. 2017:8:127-34.

12. Bustanji Y, Al-Masri IM, Qasem, A, Al-Bakri, AG, Taha MO. In silico screening for non-nucleoside hiv-1 reverse transcriptase inhibitors using physicochemical filters and high-throughput docking followed by in vitro evaluation. Chem Biol Drug. 2009;74:258-65.

Cite this article: Wijaya SJ, Yanuar A, Handayani R, Syahdi RR. In silico Analysis of Flavonoid Glycosides and its Aglycones as Reverse Transcriptase Inhibitor. Pharmacog J. 2019;11(6):1252-5. 